\title{
NADPH Oxidase Is Crucial for the Cellular Redox Homeostasis in Fungal Pathogen Botrytis cinerea
}

\author{
Hua Li,, ${ }^{1,2}$ Shiping Tian, ,2,3 and Guozheng Qin ${ }^{1,3,+}$ \\ ${ }^{1}$ Key Laboratory of Plant Resources, Institute of Botany, Chinese Academy of Sciences, Beijing 100093, China \\ ${ }^{2}$ University of Chinese Academy of Sciences, Beijing 100049, China \\ ${ }^{3}$ Key Laboratory of Post-Harvest Handing of Fruits, Ministry of Agriculture of China, Hangzhou 310021, China
}

Accepted 20 June 2019.

\begin{abstract}
During interactions, both plants and pathogens produce reactive oxygen species (ROS). Plants generate ROS for defense induction, while pathogens synthesize ROS for growth, sporulation, and virulence. NADPH oxidase (NOX) complex in the plasma membrane represents a main protein complex for ROS production in pathogens. Although NOX plays a crucial role in pathogenicity of pathogens, the underlying molecular mechanisms of NOX, especially the proteins regulated by NOX, remain largely unknown. Here, we applied an iodoacetyl tandem mass tag-based redox proteomic assay to investigate the protein redox dynamics in deletion mutant of bcnox $R$, which encodes a regulatory subunit of NOX in the fungal pathogen Botrytis cinerea. In total, 214 unique peptidyl cysteine (Cys) thiols from 168 proteins were identified and quantified in both the wild type and $\Delta b c n o x R$ mutant. The Cys thiols in the $\Delta b c n o x R$ mutant were generally more oxidized than those in the wild type, suggesting that $B c N o x R$ is essential for maintaining the equilibrium of the redox state in $B$. cinerea. Site-specific thiol oxidation analysis indicated that 142 peptides containing the oxidized thiols changed abundance significantly in the $\Delta b c n o x R$ mutant. Proteins containing these differential peptides are classified into various functional categories. Functional analysis revealed that one of these proteins, 6-phosphate dehydrogenase, played roles in oxidative stress response and pathogenesis of $B$. cinerea. These results provide insight into the potential target proteins and the ROS signal transduction pathway regulated by NOX.
\end{abstract}

Keywords: mechanisms of pathogenicity, proteomics

Botrytis cinerea is a notorious necrotrophic pathogen that infects more than 200 crop species, including almost all economically important vegetables and fruit crops, during their cultivation, storage, and distribution (Weiberg et al. 2013; Williamson et al. 2007). It causes economic losses of approximately $\$ 10$ billion to $\$ 100$ billion worldwide each year, and

${ }^{\dagger}$ Corresponding author: G. Qin; gzqin@ibcas.ac.cn

Funding: This work was supported by the National Key Research and Development Program (2018YFD1000200) and the National Natural Science Foundation of China (grant numbers 31530057 and 31172004).

*The $\boldsymbol{e}$-Xtra logo stands for "electronic extra" and indicates that one supplementary figure and two supplementary tables are published online.

The author(s) declare no conflict of interest.

(c) 2019 The American Phytopathological Society has been classified as the second most scientifically and economically important plant pathogen (Dean et al. 2012). Due to its importance in agricultural production, numerous studies have been carried out to unravel the pathogenic mechanism of this resilient fungus.

Reactive oxygen species (ROS) have been shown to play crucial roles in fungal virulence. At the early stage of pathogen infection, ROS are generated by the pathogen as well as the host and play dual roles in host-microbe interaction. ROS can damage macro-molecules and also act as signal molecules (Heller and Tudzynski 2011; Tian et al. 2013). As significant second messengers, the primary function of ROS is to regulate the cellular redox state and the downstream signal transduction (An et al. 2015; Heller and Tudzynski 2011). Similar to mammals, the primary source for ROS generation in filamentous fungi is the NADPH oxidase (NOX), which is a multisubunit complex (Lambeth 2004; O'Brien et al. 2012). The most well-characterized NOX in mammals is the phagocytic oxidase gp91 ${ }^{\text {phox }}$, which is involved in the oxidative burst defense response of neutrophils (Lambeth 2004). The gp91 ${ }^{\text {phox }}$ is a transmembrane catalytic subunit stabilized by $\mathrm{p} 22^{\mathrm{phox}}$ and its activation requires the assembly of the cytosolic subunits $\mathrm{p} 67^{\text {phox }}$, $\mathrm{p} 40^{\text {phox }}$, and $\mathrm{p} 47^{\text {phox }}$, and the small GTPase Rac (Babior et al. 2002; Diekmann et al. 1994). Fungal NoxA (Nox1) and NoxB (Nox2) are homologs of mammalian gp91 ${ }^{\text {phox }}$. They both play crucial roles in the virulence of B. cinerea, Magnaporthe grisea, Sclerotinia sclerotiorum, Fusarium graminearum, and Alternaria alternate (Egan et al. 2007; Kim et al. 2011; Segmüller et al. 2008; Wang et al. 2014; Yang and Chung 2012). Moreover, NoxA also functioned in formation of fruiting bodies in Aspergillus nidulans, Neurospora crassa, and Podospora anserina (CanoDomínguez et al. 2008; Lara-Ortíz et al. 2003; Malagnac et al. 2004), and sclerotia formation in B. cinerea and Claviceps purpurea (Giesbert et al. 2008; Segmüller et al. 2008), while NoxB was involved in ascospore germination of $N$. crassa and $P$. anserina (Cano-Domínguez et al. 2008; Malagnac et al. 2004). NoxD in B. cinerea and Pro41 in Sordaria macrospora are homologs of mammalian gp $22^{\text {phox }}$ and are involved in fruiting body maturation (Lacaze et al. 2015; Nowrousian et al. 2007). In addition, other subunits of NOX such as RAC, Rho3, and Iqg1 have also been reported to play important roles in the growth and conidia production of fungi (An et al. 2015; Marschall and Tudzynski 2016; Minz Dub et al. 2013). Among all the subunits of NOX identified in B. cinerea, the regulatory subunit BcNoxR was found to be essential because deletion of bcnox $R$ resulted in a deficiency in sclerotia formation and reduced pathogenicity (Segmüller et al. 2008). Although NOX has been demonstrated to play significant roles in the growth, differentiation, and virulence of fungal pathogens, the mechanisms by which NOX regulates 
these processes, especially the target proteins of NOX, remain largely unknown. Considering that NOX is a ROS-generating enzyme, it may function through altering the redox state of key enzymes or proteins. Exploring the protein redox dynamics might provide a novel insight into the potential target proteins and the ROS signal transduction pathway regulated by NOX.

Protein redox dynamics can be detected by redox proteomics, which monitors the redox state of cysteine (Cys) thiols. Protein thiols are significant redox switches in cells and provide flexible regulatory mechanisms for protein function. The activity of some enzymes can be switched on or off simply by oxidation of the Cys residues in the active site. Oxidation of the catalytic Cys residues of protein-tyrosine phosphatases has been reported to block the ability of this enzyme to dephosphorylate its targets (den Hertog et al. 2005). In addition to being on and off switches, the activity of many Cys-containing proteins can be regulated in a rheostat mode. Oxidation of the thiols on the 51and $75-\mathrm{kDa}$ subunits of liver mitochondrial complex I increased superoxide production, whereas reduction of these thiols reduced the superoxide production back to a basal level (Taylor et al. 2003). In addition to regulating the activity of key enzymes, reversible protein thiol oxidation also functions in transcription and transport (Dalle-Donne et al. 2003; Kuge et al. 1998).

In this study, an iodoacetyl tandem mass tag (iodoTMT)based redox proteomic assay was applied to investigate the protein redox dynamics in the $\Delta b c n o x R$ mutant in $B$. cinerea. The iodoTMT reagent is composed of a sulfhydryl-reactive iodoacetyl group for irreversible thiol labeling, a mass spectrometry (MS)-neutral spacer arm, and a tandem mass spectrometry (MS/MS) reporter for MS quantitation. To selectively analyze the thiol-labeled peptides, the sample was enriched by anti-TMT antibody resin before MS analysis. The iodoTMT approach is suitable for selective labeling and multiplex quantitation of Cys thiols in complex biological samples, and has been used to reveal light and dark modulation of thiol oxidation in many key biological processes of Cyanobacteria (Guo et al. 2014). Through the iodoTMT approach, the changes in redox states of proteins in the $\Delta b c n o x R$ mutant could be elucidated, and these proteins may represent the direct targets of NOX. In total, 214 unique peptidyl Cys thiols (thiolcontaining peptides) from 168 proteins were identified in both the wild type (WT) and the $\Delta b c n o x R$ mutant. Furthermore, we identified 142 peptides containing the oxidized thiols whose abundance changed significantly in the $\Delta b c n o x R$ mutant. Proteins containing these differential peptides were mainly found in the functional categories of carbohydrate metabolism, transcription, and translation. One of these proteins, 6-phosphate dehydrogenase (BcPGD), was found to be involved in oxidative stress response and pathogenesis of $B$. cinerea. These results indicate that BcNoxR may function through altering the redox state of various proteins containing the Cys thiols.

\section{RESULTS}

\section{Quantitative analysis of thiol oxidation in B. cinerea.}

A redox proteomic assay based on site-specific labeling of redox-modified Cys residues has been used to unravel significant biological mechanisms (Balmant et al. 2015). Here, we employed iodoTMT labeling combined with an anti-TMT resin enrichment strategy to investigate the redox state of Cys thiols in the WT and $\Delta b c n o x R$ mutant in B. cinerea (Fig. 1A). For this purpose, the oxidized thiols and total thiols were first quantified. Proteins were extracted using trichloroacetic acid (TCA) precipitation to immediately trap all of the free thiols and avoid further oxidation. To quantify oxidized thiols, the free thiols were blocked with iodoacetamide (IAM) and then the oxidized thiols were reduced to free thiols by tris(2-carboxyethyl) phosphine hydrochloride (TCEP). The newly developed free thiols were then labeled with iodoTMT reagent (as described in Materials and Methods). For total thiol quantification, samples were processed just as oxidized thiol measurements without IAM blocking. Proteins were digested with trypsin and the resulting peptides were then specifically enriched with anti-TMT resin based on an antigen antibody reaction. The peptides labeled with iodoTMT reagent were then subjected to liquid chromatography (LC)-MS/MS analysis (Supplementary Fig. S1).

The iodoTMT-labeled peptides with $P$ values $<0.05$ in both the WT and $\Delta b c n o x R$ mutant were selected for analysis. In total, 327 peptidyl Cys thiols (thiol-containing peptides) from 253 proteins were identified in the first biological replication, and 474 thiol-containing peptides from 365 proteins were identified in the second biological replication (Fig. 1B). At the intersection of the two biological replications, 214 unique thiolcontaining peptides from 168 proteins were identified and quantified in both the WT and $\Delta b c n o x R$ mutant (Fig. 1C).

\section{Redox state of Cys thiols changes in the $\Delta b c n o x R$ mutant.}

The redox state of Cys thiols in the WT and $\Delta b c n o x R$ mutant was calculated by the oxidation ratio (oxidized/total thiols). A given Cys residue might exist in three states: namely, absolutely oxidized state (oxidized/total thiols $\geq 1$ ), redox state $(0<$ oxidized/total thiols $<1)$, and absolutely reduced state (oxidized/total thiols $=0$, total thiols $>0$ ). The majority of Cys thiols ( $86 \%$ in the WT and $88 \%$ in the $\Delta b c n o x R$ mutant) were identified in redox state (Fig. 2A). Although 14\% of Cys thiols in the WT were identified in absolutely reduced state, $11 \%$ of Cys thiols in the $\Delta b c n o x R$ mutant were identified in absolutely oxidized state. The distribution of oxidation ratio of the redox Cys thiols is shown in Figure 2B, and the distribution of the oxidation ratio of all the Cys thiols is displayed in Figure 3C. The Cys thiols in the $\Delta b c n o x R$ mutant were more oxidized than those in the WT (Fig. 2B and C). Approximately $60 \%$ of Cys thiols in the WT were highly reduced, with an oxidation ratio $<0.2$, and $<3 \%$ of Cys thiols in the WT were oxidized with an oxidation ratio $>0.8$. By comparison, the oxidation ratios $<0.2$ and $>0.8$ in the $\Delta$ bcnoxR mutant were 19.16 and $18.22 \%$, respectively. These results suggest that most of the Cys thiols in the WT are in reduced state under physiological conditions, and that BcNoxR is crucial for maintaining the equilibrium of the redox state in $B$. cinerea.

\section{BcNoxR influences thiol oxidation of hundreds of proteins.}

The redox state of thiols provides the overall redox difference between the WT and $\Delta$ bcnoxR mutant. We next compared the site-specific oxidized thiols between the WT and $\Delta b c n o x R$ mutant (oxidized thiols in $\Delta b c n o x R /$ oxidized thiols in WT), and the peptides containing the oxidized thiols were identified. In the two biological replications, 267 and 364 peptides containing the oxidized thiols from 212 and 291 proteins, respectively, were separately identified whose abundance changed significantly in the $\Delta$ bcnoxR mutant according to Student's $t$ test $(P<$ 0.05 ) (Fig. 3A). In total, 152 differential peptides containing the oxidized thiols from 122 proteins were identified in both replications (Fig. 3B; Supplementary Table S2). The abundance of peptides containing the oxidized thiols might be influenced by the total protein abundance. To evaluate whether the changes in peptides containing the oxidized thiols in the $\Delta b c n o x R$ mutant were caused by the alteration of protein abundance, we carried out an isobaric tags for relative and absolute quantitation (iTRAQ)-based proteomic analysis. The abundance of proteins was used to normalize the result of the redox proteomic assay. In total, 10 peptides containing the oxidized thiols were eliminated from the differential peptides after normalization (Fig. 
3A and $\mathrm{B})$. Together, these results demonstrate that BcNoxR affects thiol oxidation of specific proteins in $B$. cinerea.

\section{BcNoxR affects thiol oxidation of proteins} with various functions.

To analyze the function of proteins with differential peptides containing the oxidized thiols, functional categorization according to Gene Ontology and KEGG pathway information was performed. These proteins were classified into 12 functional categories: namely, lipid metabolism, protein and amino acid metabolism, nucleotide metabolism, carbohydrate metabolism, signal transduction, translation, oxidation-reduction, heat shock protein, transport, transcription, cytoskeleton, and other functions. Most of the proteins were found in oxidationreduction, carbohydrate metabolism, and translation (Fig. 4). These results suggest that BcNoxR can influence the redox state of proteins with various functions.

\section{Functional associations of redox-sensitive proteins.}

To gain an overall insight into the pathways that are potentially redox regulated by BcNoxR and the relationship of the redox-sensitive proteins influenced by $\mathrm{BcNoxR}$, proteins with differential peptides containing the oxidized thiols were submitted to the String database for interaction analysis. Each protein has a new identifier (EDN number) in String data, and the corresponding identifiers (Bcin number) were from the EnsemblFungi $B$. cinerea genome database. The redoxsensitive proteins influenced by BcNoxR were involved in different biological pathways and interact with proteins with relevant function (Fig. 5). Stronger associations between proteins are represented by thicker lines, and several key biological pathways can be observed, including transcription and translation in the lower left quarter, as well as carbohydrate metabolism in the middle of the map (Fig. 5).

\section{BcPGD is essential for oxidative stress response and pathogenesis of $B$. cinerea.}

In our redox proteomic analysis, we found that BcPGD, an enzyme participating in the pentose-phosphate pathway and involved in NADPH generation, showed differential peptides that contain the oxidized thiols between the WT and $\Delta b c n o x R$ mutant. The transcript level of bcpgd has been previously demonstrated to be affected by BcNoxR ( $\mathrm{Li}$ et al. 2016). BcPGD exhibited higher oxidation levels in the $\Delta b c n o x R$ mutant, indicating that the redox state of BcPGD was influenced by $\mathrm{BcNoxR}$. To investigate whether $\mathrm{BcNoxR}$ functions by targeting BcPGD, deletion mutants of bcpgd were generated, and the oxidative stress response and virulence of $\Delta b c p g d$ mutants were assayed. Both the $\Delta b c p g d$ and $\Delta b c n o x R$ mutants were sensitive to oxidative stress (Fig. 6A). The virulence of these two mutants on grape was reduced when compared with the WT (Fig. 6B). Complementation of bcpgd or bcnoxR in the mutants restored the sensitivity to oxidative stress and the virulence. These results indicated that $\mathrm{BcNoxR}$ might regulate the oxidative stress response and pathogenesis, at least partially, by affecting the oxidation state of BcPGD.

\section{DISCUSSION}

In the present study, we applied a recently developed redox proteomic approach with iodoTMT labeling to investigate the changes in the redox state of Cys thiols in the $\Delta b c n o x R$ mutant
A

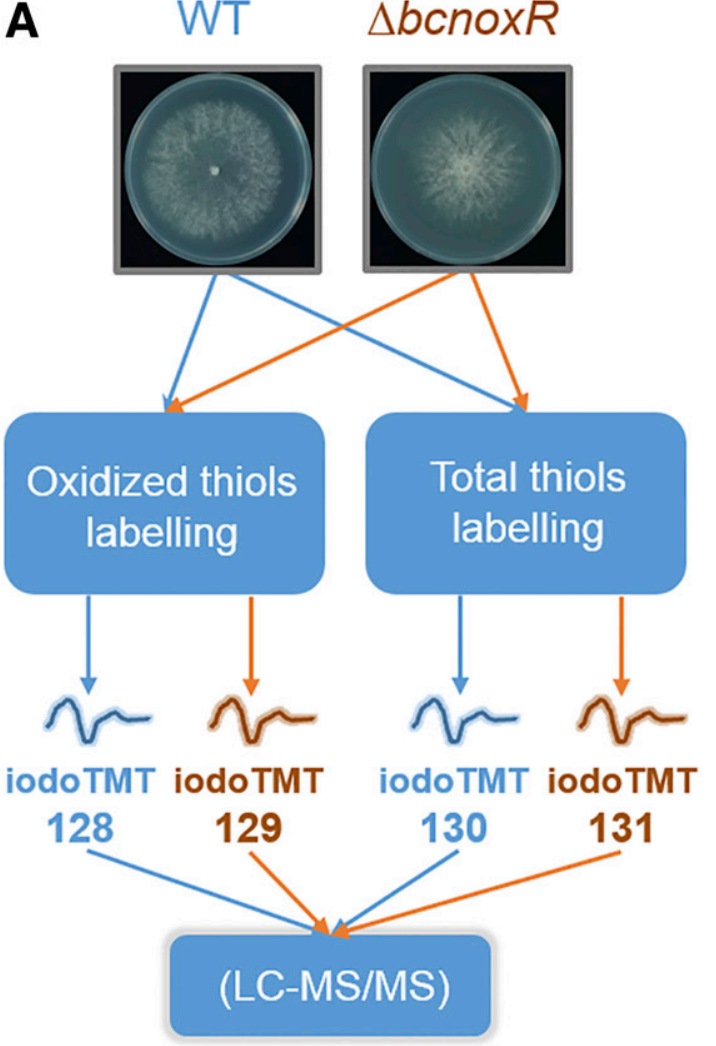

B

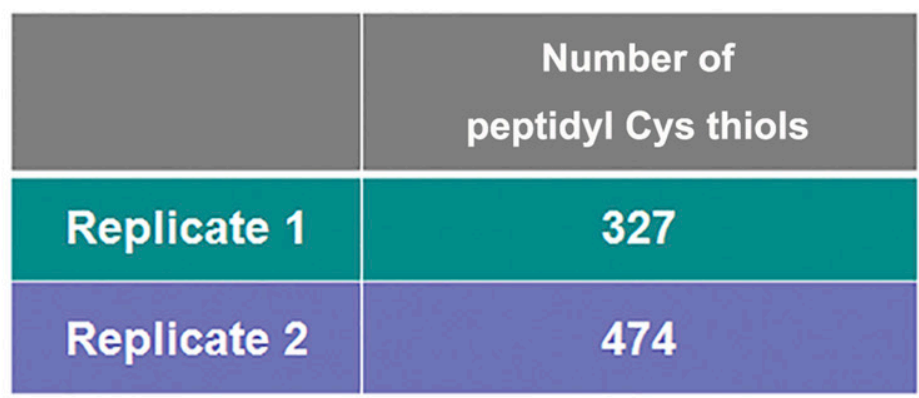

\section{Peptidyl Cys thiols}

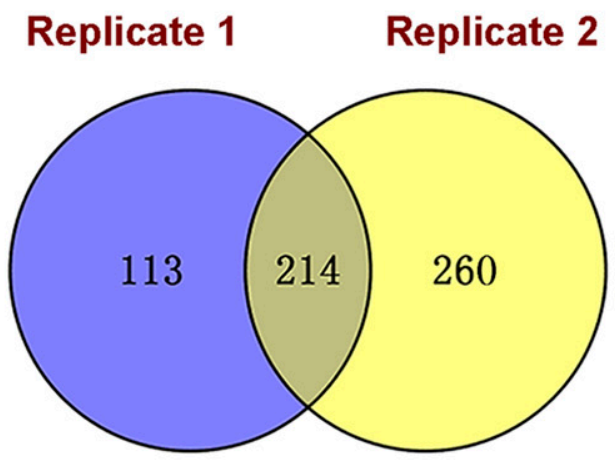

Fig. 1. Peptidyl cysteine (Cys) thiols identified in the iodoacetyl tandem mass tag (iodoTMT)-based redox proteomics. A, The overall workflow scheme for protein thiol redox proteomics in this study. Proteins of the wild type (WT) and $\Delta b c n o x R$ mutant were extracted, and the oxidized thiols and total thiols of each strain were labeled with iodoTMT128/130 and iodoTMT129/131, respectively. LC-MS/MS = liquid chromatography-tandem mass spectrometry. B, Table of peptidyl Cys thiols identified in the two biological replications. C, Venn diagram of peptidyl Cys thiols identified in the two biological replications. 
in $B$. cinerea. The BcNoxR protein is the regulatory subunit of NOX, which is a multiple-subunit complex that generates ROS as its primary function, and has been demonstrated to participate in growth and sporulation as well as virulence in $B$. cinerea (Segmüller et al. 2008). Exploring the changes in redox state of Cys thiols in the $\Delta b c n o x R$ mutant would provide a broad insight into the signaling pathway and proteins regulated by NOX.

The iodoTMT consists of a Cys reactive group, a mass normalizer, and a mass report group. The Cys reactive group irreversibly labels free thiols on Cys residues. It is challenging to label the redox state of Cys thiols at physiological conditions because free thiols are prone to oxidation during sample preparation processing. In our approach, free thiols were trapped by TCA precipitation to retain their redox state in vivo and avoid artificial thiol oxidation during sample preparation. To selectively analyze the Cys thiols and reduce sample complexity, the sample was effectively enriched with the Thermo Scientific immobilized anti-TMT antibody resin. In total, 214 peptidyl Cys thiols from 168 proteins were identified to be labeled with iodoTMT (Fig. 1; Supplementary Table S1). Most of the peptidyl Cys thiols were in a reduced state, perhaps due to the overall intracellular reduced environment (Fig. 2). It is noteworthy that the redox state of peptidyl Cys thiols was more oxidized in the $\Delta b c n o x R$ mutant. This is unexpected because BcNoxR is the regulatory subunit of NOX, which generates ROS as its primary function. The peptidyl Cys thiols in the $\Delta b c n o x R$ mutant are supposed to be more reduced rather than oxidized. However, this is consistent with previous research showing that $\Delta b c n o x$ mutants are not significantly impaired in ROS production and that the catalytic subunits of BcNox are responsible for normal tolerance to oxidative stress caused by
$\mathrm{H}_{2} \mathrm{O}_{2}$ (Segmüller et al. 2008). In addition to the difference in redox state between the WT and $\Delta b c n o x R$ mutant, the sitespecific identification and quantification of oxidized Cys thiols were also investigated. After multiplex labeling and effective enrichment of the iodoTMT, 152 peptides containing the
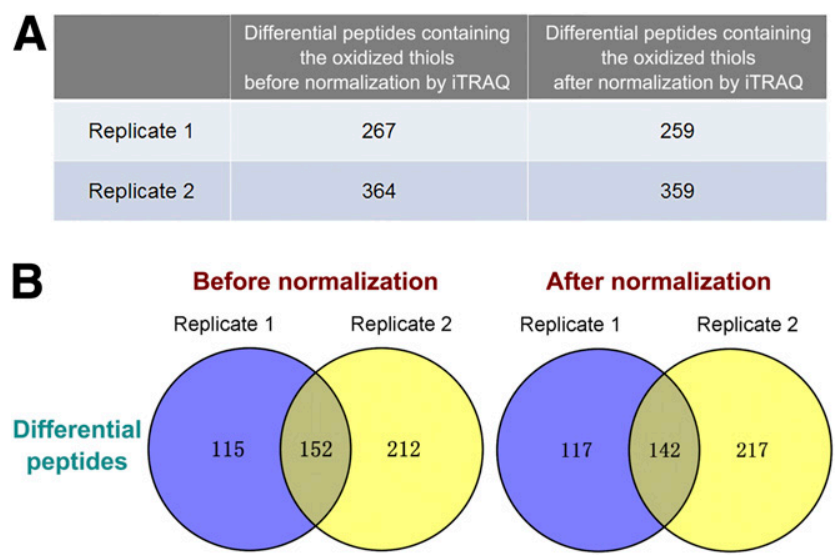

Fig. 3. Site-specific thiol oxidation in the wild type (WT) and $\Delta b c n o x R$ mutant. A, Table of differential peptides containing the oxidized thiols identified in the two biological replications. B, Venn diagram of differential peptides containing the oxidized thiols in the two biological replications. To exclude the influence of changes in protein abundance on oxidized peptidyl cysteine (Cys) quantification, an isobaric tags for relative and absolute quantitation (iTRAQ)-based proteomic approach was conducted. Only proteins identified below $1 \%$ global false discovery rate and with a $P$ value $<0.05$ were used to normalize the result of thiol oxidation.
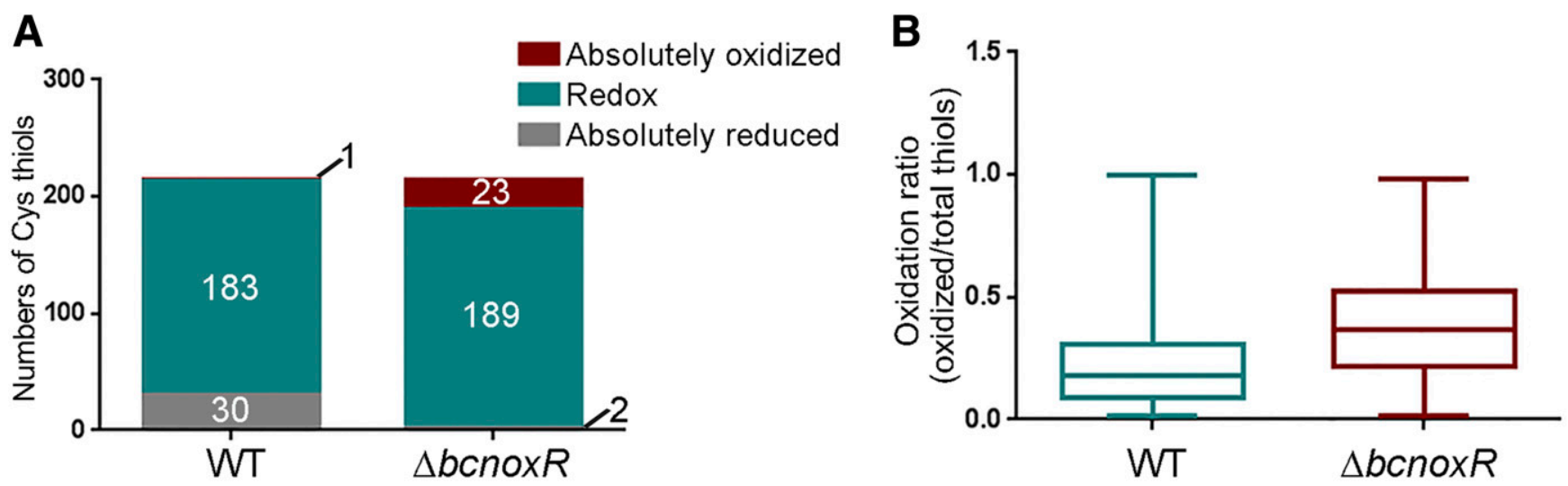

C
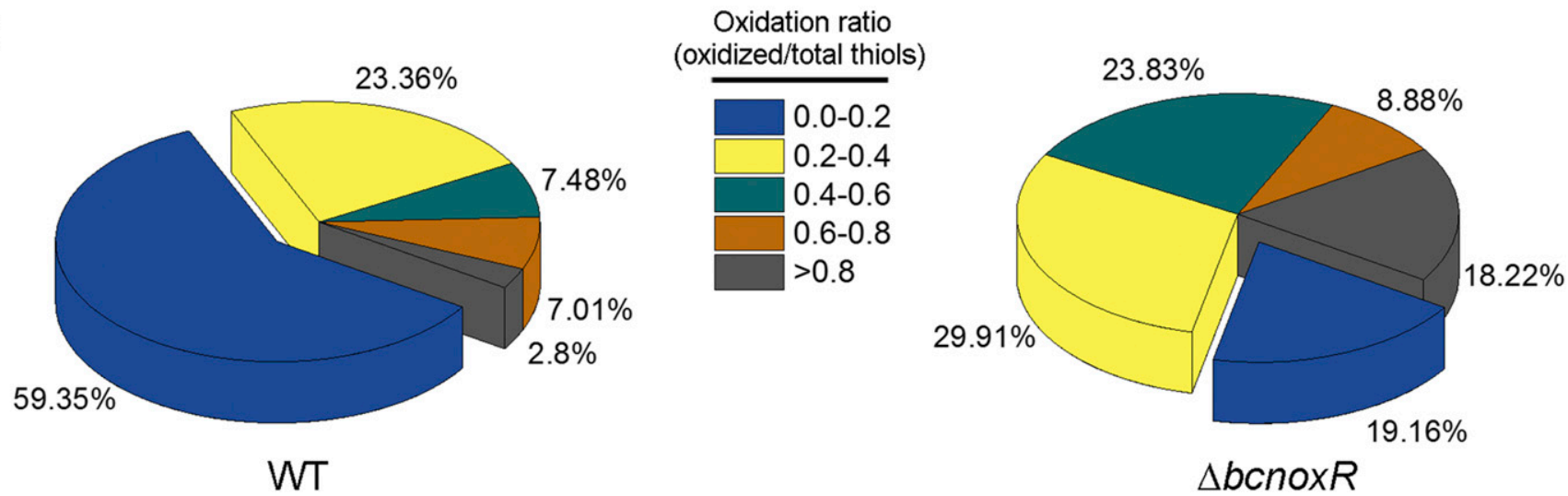

Fig. 2. Oxidation state of cysteine (Cys) thiols in the wild type (WT) and $\Delta b c n o x R$ mutant. A, Overview of the redox state of Cys thiols in the WT and $\Delta b c n o x R$ mutant. B, Box-plot showing the oxidation state of Cys thiols in the WT and $\Delta$ bcnoxR mutant. C, Distribution of oxidation state of Cys thiols in the WT and $\Delta$ bcnoxR mutant. 
oxidized thiols were found to change abundance significantly in the $\Delta b c n o x R$ mutant (Fig. 3). Our previous study has shown that deletion of bcnoxR affected the expression of 49 proteins in $B$. cinerea ( $\mathrm{Li}$ et al. 2016). Therefore, the difference in peptides that contain the oxidized thiols might be caused by the alteration of protein abundance in the $\Delta b c n o x R$ mutant. We conducted an iTRAQ-based proteomic analysis to determine whether the changes in abundance of peptides containing the oxidized thiols were due to the alteration of protein abundance. The result indicated that 142 of the 152 peptides containing the oxidized thiols changed abundance significantly in the $\Delta$ bcnoxR mutant independent of the protein abundance (Fig. 3).

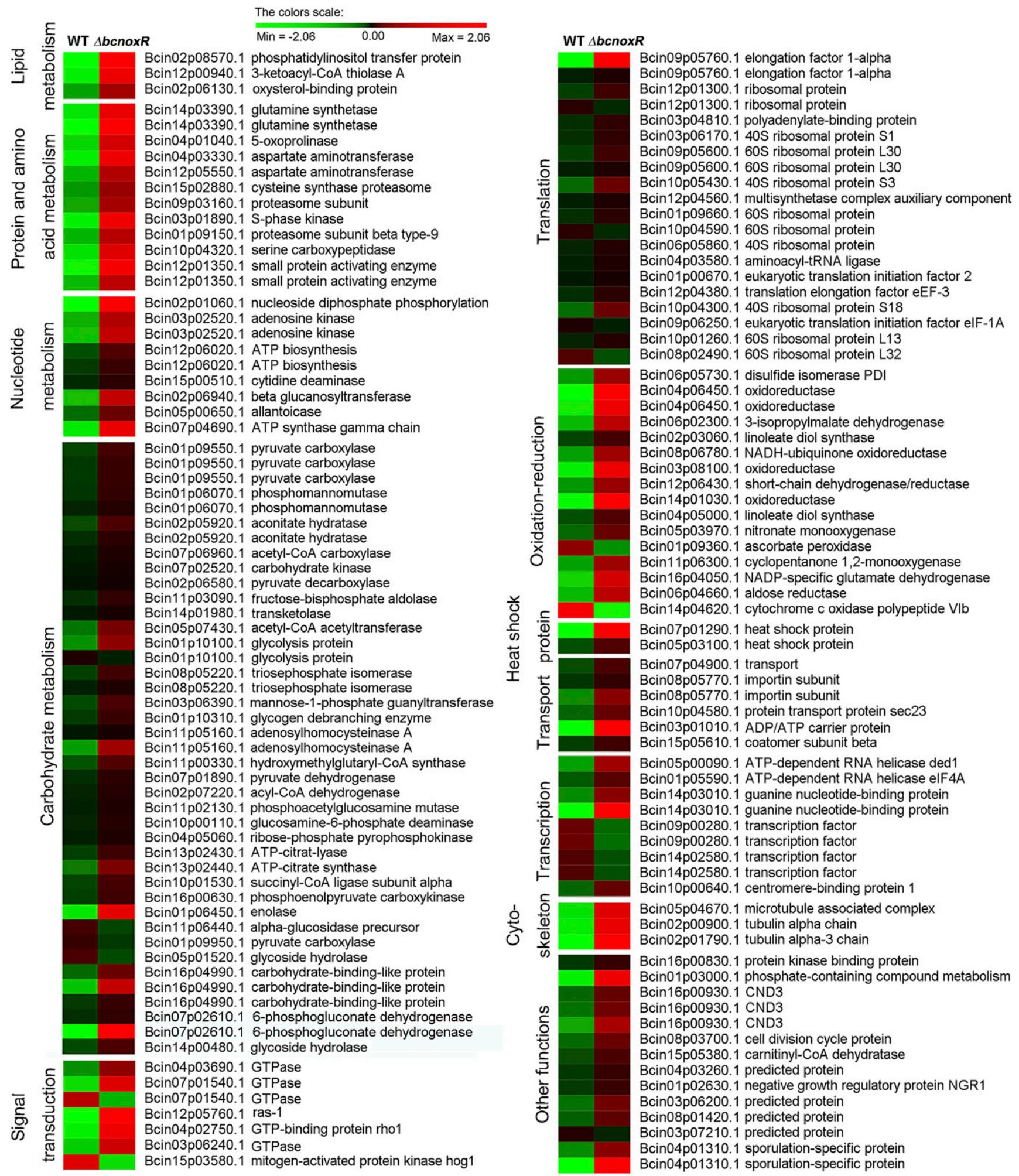

Fig. 4. Quantitative analysis of thiol oxidation in the wild type (WT) and $\Delta b c n o x R$ mutant. Proteins with the differential peptides, which contain the oxidized thiols, were categorized according to Gene Ontology and KEGG pathway information. Each row indicates a peptide containing the oxidized thiols, and the column represents WT and $\Delta b c n o x R$ mutant; Min $=$ minimum and Max $=$ maximum. Protein identifiers (Bcin numbers) and functions are shown. Red indicates peptides with higher abundance of oxidized cysteine (Cys) thiols, while green indicates peptides with lower abundance of oxidized Cys thiols. 
Proteins with differential peptides that contain the oxidized thiols in the $\Delta b c n o x R$ mutant are classified into various functional categories such as oxidation-reduction, carbohydrate metabolism, transcription, and translation (Fig. 4). Fifteen proteins, which contain 16 of these differential peptides, function in oxidation-reduction, including disulfide isomerase, NADH-ubiquinone oxidoreductase, NADP-specific glutamate dehydrogenase, and so on. Disulfide isomerase plays important roles in the refolding of denatured RNase A, and maturation of secretory proteins in A. niger, a common contaminant of food (Ngiam et al. 2000). NADH-ubiquinone oxidoreductase is an energy-transducing enzyme coupling NADH oxidation with ubiquinone reduction and is involved in the generation of a proton gradient for further ATP synthesis. Disruption of the related gene in $N$. crassa resulted in growth impediment of this mold (Fecke et al. 1994).

Thirty-one proteins that contain 41 differential peptides were found to participate in carbohydrate metabolism, including the TCA cycle, glycolysis/gluconeogenesis, as well as the pentosephosphate pathway (Fig. 4). Proteins that participate in the TCA cycle includes aconitate hydratase, ATP-citrate synthase, and succinyl-CoA ligase. The TCA cycle has been reported to tune the redox state of cells (Cardaci and Ciriolo 2012). In this study, the redox state of enzymes in the TCA cycle was found to be affected in the $\Delta b c n o x R$ mutant. These proteins may function in the downstream signaling pathway of BcNoxR. Protein thiol redox was supposed to be the regulator of enzyme activity in gluconeogenesis/glycolysis (Hui et al. 2004). The thiol redox state of five proteins in glycolysis/gluconeogenesis (namely, fructose-bisphosphate aldolase, glycolysis protein, triosephosphate isomerase, phosphoenolpyruvate carboxykinase, and enolase) were identified in the $\Delta b c n o x R$ mutant. The activity of these proteins may be regulated by $\mathrm{BcNoxR}$ through a cellular redox state. In addition, transketolase, which functions in the pentosephosphate pathway, was identified. The pentose-phosphate pathway has been demonstrated to be a metabolic redox sensor and has the ability to regulate transcription during the antioxidant response (Krüger et al. 2011). BcPGD, an enzyme participating in the pentose-phosphate pathway, was ascertained to exhibit an increased oxidative level in the $\Delta b c n o x R$ mutant. The $\Delta b c p g d$ mutants are more susceptible to oxidative stress and show reduced virulence (Fig. 6). It is likely that BcNoxR also functions through altering the redox state of key enzymes in the pentose-phosphate pathway.

Several proteins involved in transcription and translation were identified in the redox proteomics, including two transcription

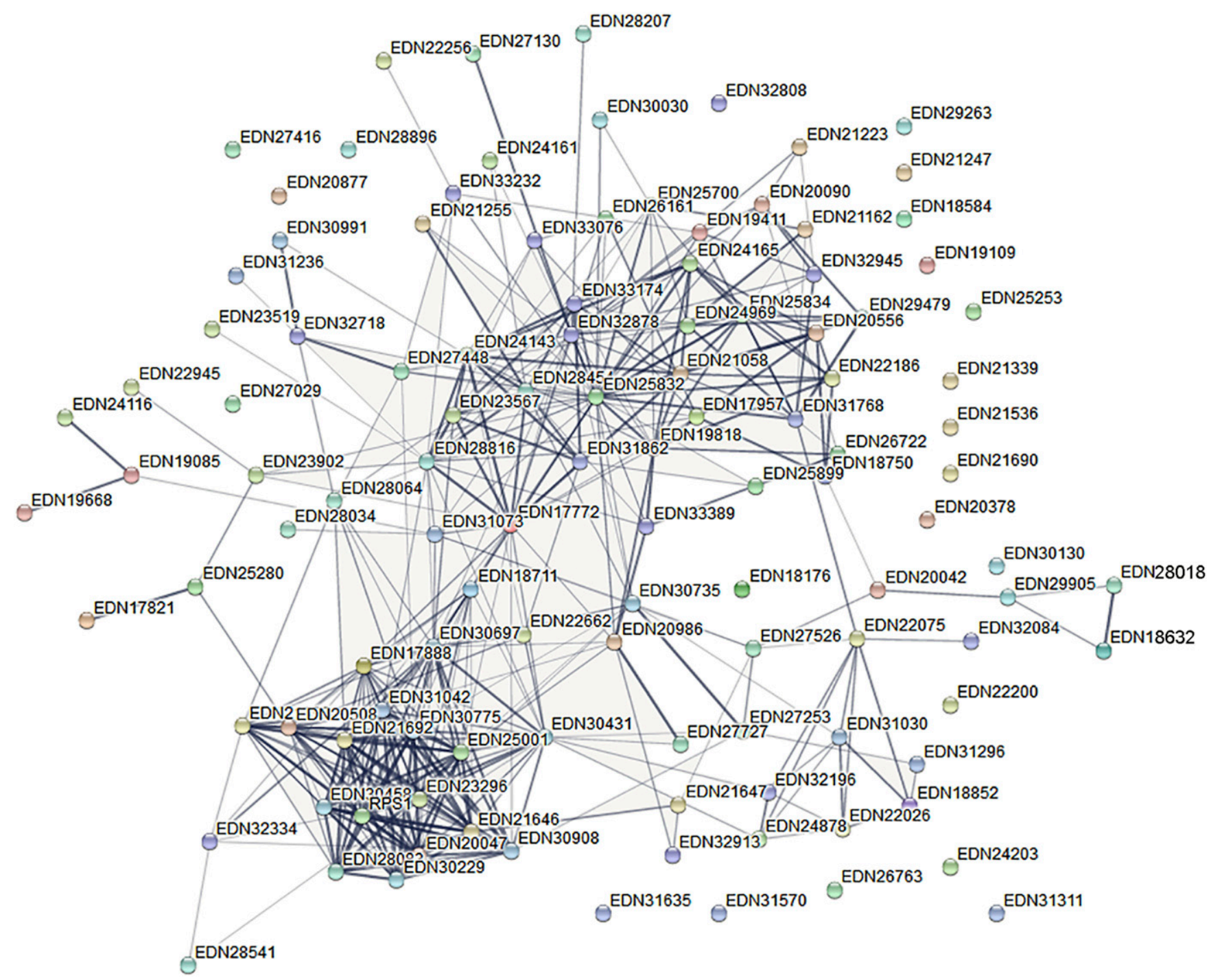

Fig. 5. Functional association analysis of proteins with differential peptides containing the oxidized thiols between the wild type (WT) and $\Delta b c n o x R$ mutant using the String database. Proteins with differential peptides, which contain the oxidized thiols, were blasted against the Botrytis cinerea database from Broad Institute, and the corresponding gene identifiers (BC1G numbers) were used for String database analysis. Thicker lines represent stronger data support. 
factors and proteins associated with translation initiation and elongation. The redox state and ROS signaling have been widely reported to regulate gene transcription and translation (Foyer and Noctor 2003). BcNoxR might affect the growth and virulence of $B$. cinerea through influencing transcription or translation of key proteins.

In conclusion, we applied a redox proteomic approach (iodoTMT labeling coupled with anti-TMT resin enrichment) to investigate the changes in the thiol redox state in the $\Delta b c n o x R$ mutant in B. cinerea, and found that $\mathrm{BcNoxR}$ is essential for the cellular redox homeostasis. We unraveled the crucial role of BcNoxR for maintaining the reduced environment of $B$. cinerea, despite its primary role in ROS generation. Our results also uncovered potential targets of BcNoxR in signal transduction pathways, including proteins participating in oxidation-reduction, carbohydrate metabolism, transcription, and translation.

\section{MATERIALS AND METHODS}

\section{Fungal strains and culture conditions.}

$B$. cinerea (B05.10) was used as the WT control, and the $\triangle b c n o x R$ mutant and bcnox ${ }^{c o m}$ were obtained in our previous research (Li et al. 2016). For $\Delta b c p g d$ mutant construction, $1 \mathrm{~kb}$ of a flanking sequence of the bcpgd gene was amplified and ligated into pLOB7 vector to flank the HPH marker to construct the replacement vectors. The replacement vectors were amplified and introduced into the protoplasts of B05.10. Transformants were selected on hygromycin and confirmed by PCR. For $\Delta b c p g d$ complementary strain construction, the complete target gene, with approximately $2 \mathrm{~kb}$ of upstream sequences and $500 \mathrm{bp}$ of downstream sequences, was amplified and cloned into the pNAN-OGG vector. The constructed vectors were amplified and introduced into the protoplasts of $\Delta b c p g d$ mutants. The WT and $\Delta b c n o x R$ and $\Delta b c p g d$ mutants were normally cultured at $22^{\circ} \mathrm{C}$ on complete medium containing glucose at $10 \mathrm{~g} \mathrm{liter}^{-1}$, peptone at $2 \mathrm{~g} \mathrm{liter}^{-1}$, yeast extract at $1 \mathrm{~g}$ liter ${ }^{-1}$, acid hydrolyzed casein at $1 \mathrm{~g} \mathrm{liter}^{-1}, \mathrm{NaNO}_{3}$ at $6 \mathrm{~g}$ liter $^{-1}, \mathrm{KCl}$ at $0.5 \mathrm{~g} \mathrm{liter}^{-1} \mathrm{MgSO}_{4} \cdot 7 \mathrm{H}_{2} \mathrm{O}$ at $1.11 \mathrm{~g} \mathrm{liter}^{-1}$, $\mathrm{KH}_{2} \mathrm{PO}_{4}$ at $1.5 \mathrm{~g} \mathrm{liter}^{-1}$, and yeast nitrogen base at $0.05 \mathrm{~g} \mathrm{liter}^{-1}$. Potato dextrose broth (PDB) was used to culture $B$. cinerea for protein extraction.

\section{Sample preparation.}

Conidia with an initial concentration of the WT and $\Delta b c n o x R$ mutant of $5 \times 10^{4}$ spores $/ \mathrm{ml}$ were cultured in PDB. After $48 \mathrm{~h}$ of culture, the mycelia were collected and rinsed twice with $\mathrm{KC}$ buffer $(0.6 \mathrm{M} \mathrm{KCl}$ and $50 \mathrm{mM} \mathrm{CaCl} 2)$. The mycelia were then ground into a fine powder using liquid nitrogen and resuspended with $20 \%$ (wt/vol) TCA. The homogenate was incubated on ice for $20 \mathrm{~min}$ and centrifuged at $15,000 \times g$ for $30 \mathrm{~min}$ to precipitate proteins. The precipitates were washed with ice-cold $20 \%$ (wt/vol) TCA and acetone to remove the majority of TCA. The proteins were solubilized in $300 \mu \mathrm{l}$ of HES buffer (50 mM HEPES [pH 8.0], $1 \mathrm{mM}$ EDTA, and $0.1 \%$ sodium dodecyl sulfate), and a BCA protein assay kit (Thermo Scientific Pierce) was used to determine protein content.

\section{Quantitative redox proteomics.}

For oxidized thiol labeling, $100 \mu \mathrm{g}$ of protein $(1 \mu \mathrm{g} / \mu \mathrm{l})$ of the WT or $\Delta b c n o x R$ mutant was alkylated by $200 \mathrm{mM}$ IAM for $30 \mathrm{~min}$ at room temperature to block free thiols. To remove excessive IAM, the proteins were precipitated with $600 \mu \mathrm{l}$ of prechilled acetone overnight at $-20^{\circ} \mathrm{C}$. After centrifugation at $10,000 \times g$ for $10 \mathrm{~min}$ at $4^{\circ} \mathrm{C}$, the pellet was air dried for $10 \mathrm{~min}$ and dissolved in $100 \mu \mathrm{l}$ of HES buffer. TCEP was added to each protein sample with a final concentration of $5 \mathrm{mM}$ to reduce oxidized thiols, and the protein sample was incubated at $50^{\circ} \mathrm{C}$
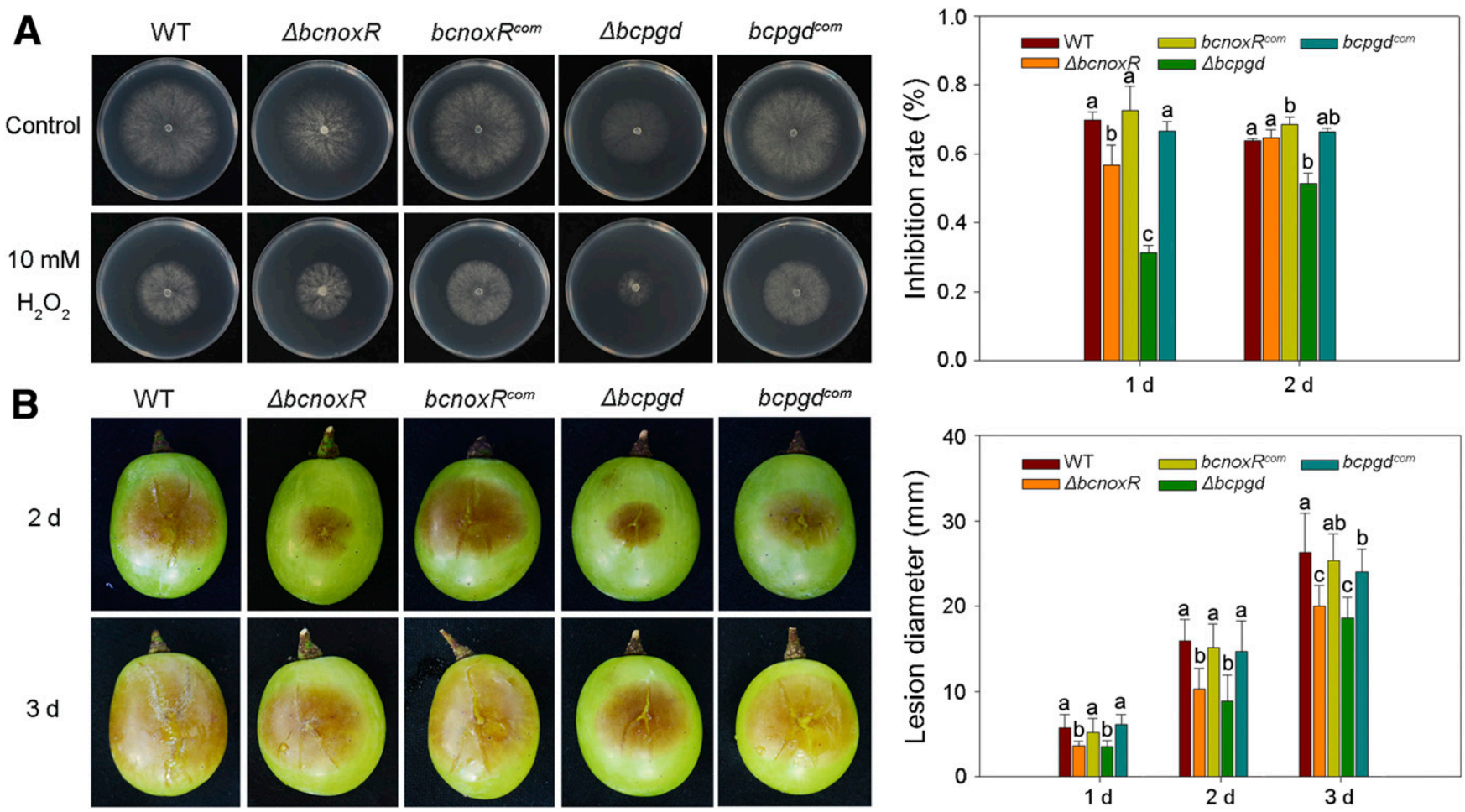

Fig. 6. Involvement of BcNoxR and BcPGD in oxidative stress response and virulence. A, Colony morphology and diameter of the wild type (WT), $\Delta b c n o x R$, bcnoxR $R^{\text {com }}, \Delta$ bcpgd, and bcpgd ${ }^{\text {com }}$ after 48 h of growth on complete medium plates with or without $10 \mathrm{mM} \mathrm{H}_{2} \mathrm{O}_{2}$. B, Representative photograph of disease symptoms and lesion diameter after infection of various strains for 2 or 3 days (d). Columns with different letters indicate significant difference at the indicated time, using the least significant difference test $(P<0.05)$. 
for $1 \mathrm{~h}$. Sufficient (a 10-fold excess of reagent over free thiol concentration) iodoTMT (Thermo Scientific) reagent $(0.2 \mathrm{mg}$ for every $100 \mu \mathrm{g}$ of protein) was added to each sample. Oxidized thiols of the WT were labeled with iodoTMT128, while oxidized thiols of the $\Delta b c n o x R$ mutant were labeled with iodoTMT129. The reaction was carried out at $37^{\circ} \mathrm{C}$ for $1 \mathrm{~h}$, protected from light to ensure an effective labeling. To quench the reaction, each protein sample was mixed with dithiothreitol at a final concentration of $20 \mathrm{mM}$ and incubated at $37^{\circ} \mathrm{C}$ for 15 min protected from light.

For total thiol labeling, a protein sample was reduced without alkylation. The procedure of thiol reduction and iodoTMT labeling was the same as for oxidized thiol labeling. Total thiols of the WT were labeled with iodoTMT130, while total thiols of the $\Delta b c n o x R$ mutant were labeled with iodoTMT131.

An equal amount of labeled sample $(100 \mu \mathrm{g})$ of the WT and $\Delta b c n o x R$ mutant were mixed and six volumes of prechilled $\left(-20^{\circ} \mathrm{C}\right)$ acetone was added into the combined sample to precipitate proteins. Acetone-precipitated protein pellets were collected by centrifugation at $10,000 \times g$ for $10 \mathrm{~min}$ at $4^{\circ} \mathrm{C}$, and dissolved with $50 \mathrm{mM}$ ammonium bicarbonate buffer $(\mathrm{pH} 8)$. The sample was then digested with $10 \mu \mathrm{g}$ of trypsin $(2.5 \mu \mathrm{g}$ of trypsin per $100 \mu \mathrm{g}$ of protein) overnight at $37^{\circ} \mathrm{C}$. After digestion, the sample was acidified by trifluoroacetic acid and peptides were cleaned up using C18 spin columns.

Labeled peptides were enriched by anti-TMT resin following the user's manual. Anti-TMT resin was washed three times with one column volume of Tris-buffered saline (TBS) (pH 7.5) before use. Peptides were lyophilized and resuspended with $100 \mu \mathrm{l}$ of TBS, and loaded onto the anti-TMT resin with endover-end mixing at $4^{\circ} \mathrm{C}$ overnight. The supernatant was removed and the resin was washed five times with one column volume of TBS with $2 \mathrm{M}$ urea, followed by three times with one column volume of TBS and water. The labeled peptides were then eluted with four column volumes of TMT elution buffer, lyophilized using a vacuum concentrator, and resuspended to generate the final sample for LC-MS/MS analysis. Two biological replications, including two technical replications each, were conducted.

\section{iTRAQ labeling.}

For protein quantification, an iTRAQ-based proteomic approach was employed. Proteins $(40 \mu \mathrm{g})$ from the WT or $\triangle b c n o x R$ mutant were reduced using TCEP with a final concentration of $10 \mathrm{mM}$ and incubated at $60^{\circ} \mathrm{C}$ for $1 \mathrm{~h}$. The samples were then alkylated by methyl methanethiosulfonate (MMTS) at a final concentration of $50 \mathrm{mM}$ for $10 \mathrm{~min}$. After alkylation, protein samples were digested with $0.8 \mu \mathrm{g}$ of trypsin (Promega Corp.) at $37^{\circ} \mathrm{C}$ overnight using the filter-aided sample preparation method (Wiśniewski et al. 2009). The tryptic peptides were collected at $11,000 \times g$ for $15 \mathrm{~min}$ at room temperature and labeled with the iTRAQ Reagents 4-plex Kit (Applied Biosystems) according to the manufacturer's protocol. Protein samples of the WT and $\Delta b c n o x R$ mutant were labeled with iTRAQ tags 116 and 117, respectively. Three independent biological replications were conducted. The iTRAQlabeled samples in each biological replication were separately pooled, lyophilized, and submitted to nanoLC-MS/MS analysis. Three biological replications were conducted.

\section{NanoLC-MS/MS.}

The MS analysis was carried out using a nanoLC system (NanoLC-2D Ultra Plus; Eksigent) equipped with a TripleTOF 5600 Plus mass spectrometer (AB SCIEX) according to previous research in our lab (Wang et al. 2017). Peptide samples were desalted with a trap column $(100 \mu \mathrm{m}$ by $20 \mathrm{~mm})$ and eluted on an analytical column $(75 \mu \mathrm{m}$ by $150 \mathrm{~mm})$ packed with
Magic C18-AQ $5 \mu \mathrm{m} 200 \AA$ phase (Michrom Bioresources, Inc). Peptides were then separated by a linear gradient of 5 to $30 \%$ mobile phase B in mobile phase A. Mobile phase A was $0.1 \%$ formic acid and $100 \%$ water, while mobile phase B was $0.1 \%$ formic acid and $100 \%$ acetonitrile. Precursor ions across the mass range of 350 to $1,500 \mathrm{~m} / \mathrm{z}$ were chosen. Twenty-five precursors per cycle from each MS spectrum were chosen for fragmentation to the maximum. Tandem mass spectra were recorded in high-sensitivity mode with resolution $>15,000$.

\section{Database searching.}

For database searching of redox proteomics, the raw data of LCMS/MS were converted into data files using AB SCIEX MS data converter V1.3. The data file was used to search against the $B$. cinerea protein database (Botrytis_cinerea_ASM83294v1.pep.all.fasta) with mascot 2.4.0. The key search parameters used were (i) enzyme, trypsin; (ii) maximum missed cleavages, 1 ; (iii) peptide mass tolerance, $10 \mathrm{ppm}$; (iv) fragment mass tolerance, $0.05 \mathrm{Da}$; and (v) instrument type, ESI-QUAD-TOF (electrospray ionization quadrupole time-of-flight). Only peptides with scores $>18$ were chosen for further analysis; for individual peptides, scores $>18$ indicate identity or extensive homology $(P<0.05)$.

For database searching of iTRAQ labeling, protein identification and quantification were carried out using ProteinPilot 4.5 software (AB SCIEX). The mass spectra data were searched against the $B$. cinerea protein database with the following parameters: (i) sample type: iTRAQ 4-plex (peptide labeled), (ii) Cys alkylation: MMTS, (iii) digestion: trypsin, (iv) instrument: TripleTOF 5600, (v) species: none, (vi) quantitate: yes, (vii) bias correction: yes, (viii) background correction: yes, (ix) search effort: thorough, and (x) false discovery rate (FDR) analysis: yes. The peptide for quantification was selected by the Pro Group algorithm (AB SCIEX) automatically. Global FDR was estimated by the reverse database search method (Elias and Gygi 2007). Only proteins identified below $1 \%$ of global FDR were exported.

\section{Data analysis.}

To analyze the redox state of thiols in the WT and $\Delta b c n o x R$ mutant, the proportions of oxidized thiols and total thiols were calculated. To analyze the thiol oxidation between the WT and $\Delta b c n o x R$ mutant, the ratios of oxidized thiols were computed. An iTRAQ-based proteomic approach was applied to normalize the results of redox proteomics. The biological functions of proteins with peptidyl Cys residues that showed significant differences were clustered based on Gene Ontology and KEGG pathway information. The String database was searched for known and predicted protein-protein interactions. The Venn diagram was constructed using web-based Venn diagram software (Venny 2.1), and hierarchical clustering was generated by the PermutMatrix software (version 1.9.3).

\section{ACKNOWLEDGMENTS}

We thank P. Tudzynski (Westfaelische Wilhelms-Universitaet Muenster, Germany) for kindly providing the B. cinerea haploid strain B05.10.

\section{AUTHOR-RECOMMENDED INTERNET RESOURCES}

Broad Institute Botrytis cinerea genome database: https://www.broadinstitute.org/fungal-genome-initiative/botrytis-cinereagenome-project

EnsemblFungi Botrytis cinerea genome database: http://fungi.ensembl.org/Botrytis_cinerea/Info/Index

Matrix Science web site: www.matrixscience.com

NCBI nonredundant database: https://www.ncbi.nlm.nih.gov/

String database: https://string-db.org/cgi/input.pl?UserId=zInS9adbdUSD\& sessionId=uchKlpp8MEut\&input_page_active_form=multiple_identifiers 


\section{LITERATURE CITED}

An, B., Li, B., Qin, G., and Tian, S. 2015. Function of small GTPase Rho3 in regulating growth, conidiation and virulence of Botrytis cinerea. Fungal Genet. Biol. 75:46-55.

Babior, B. M., Lambeth, J. D., and Nauseef, W. 2002. The neutrophil NADPH oxidase. Arch. Biochem. Biophys. 397:342-344.

Balmant, K. M., Parker, J., Yoo, M. J., Zhu, N., Dufresne, C., and Chen, S. 2015. Redox proteomics of tomato in response to Pseudomonas syringae infection. Hortic. Res. 2: Article 15043.

Cano-Domínguez, N., Alvarez-Delfín, K., Hansberg, W., and Aguirre, J. 2008. NADPH oxidases NOX-1 and NOX-2 require the regulatory subunit NOR-1 to control cell differentiation and growth in Neurospora crassa. Eukaryot. Cell 7:1352-1361.

Cardaci, S., and Ciriolo, M. R. 2012. TCA cycle defects and cancer: When metabolism tunes redox state. Int. J. Cell Biol. 2012: Article 161837.

Dalle-Donne, I., Rossi, R., Giustarini, D., Colombo, R., and Milzani, A. 2003. Actin S-glutathionylation: Evidence against a thiol-disulphide exchange mechanism. Free Radic. Biol. Med. 35:1185-1193.

Dean, R., Van Kan, J. A., Pretorius, Z. A., Hammond-Kosack, K. E., Di Pietro, A., Spanu, P. D., Rudd, J. J., Dickman, M., Kahmann, R., Ellis, J., and Foster, G. D. 2012. The top 10 fungal pathogens in molecular plant pathology. Mol. Plant Pathol. 13:414-430.

den Hertog, J., Groen, A., and van der Wijk, T. 2005. Redox regulation of protein-tyrosine phosphatases. Arch. Biochem. Biophys. 434:11-15.

Diekmann, D., Abo, A., Johnston, C., Segal, A. W., and Hall, A. 1994 Interaction of Rac with p67phox and regulation of phagocytic NADPH oxidase activity. Science 265:531-533.

Egan, M. J., Wang, Z. Y., Jones, M. A., Smirnoff, N., and Talbot, N. J. 2007. Generation of reactive oxygen species by fungal NADPH oxidases is required for rice blast disease. Proc. Natl. Acad. Sci. U.S.A. 104: $11772-11777$

Elias, J. E., and Gygi, S. P. 2007. Target-decoy search strategy for increased confidence in large-scale protein identifications by mass spectrometry. Nat. Methods 4:207-214.

Fecke, W., Sled, V. D., Ohnishi, T., and Weiss, H. 1994. Disruption of the gene encoding the NADH-binding subunit of NADH: Ubiquinone oxidoreductase in Neurospora crassa. Eur. J. Biochem. 220:551-558.

Foyer, C. H., and Noctor, G. 2003. Redox sensing and signalling associated with reactive oxygen in chloroplasts, peroxisomes and mitochondria. Physiol. Plant. 119:355-364.

Giesbert, S., Schürg, T., Scheele, S., and Tudzynski, P. 2008. The NADPH oxidase Cpnox1 is required for full pathogenicity of the ergot fungus Claviceps purpurea. Mol. Plant Pathol. 9:317-327.

Guo, J., Nguyen, A. Y., Dai, Z., Su, D., Gaffrey, M. J., Moore, R. J., Jacobs, J. M., Monroe, M. E., Smith, R. D., Koppenaal, D. W., Pakrasi, H. B., and Qian, W.-J. 2014. Proteome-wide light/dark modulation of thiol oxidation in cyanobacteria revealed by quantitative site-specific redox proteomics. Mol. Cell. Proteomics 13:3270-3285.

Heller, J., and Tudzynski, P. 2011. Reactive oxygen species in phytopathogenic fungi: Signaling, development, and disease. Annu. Rev. Phytopathol. 49:369-390.

Hui, T. Y., Sheth, S. S., Diffley, J. M., Potter, D. W., Lusis, A. J., Attie, A. D., and Davis, R. A. 2004. Mice lacking thioredoxin-interacting protein provide evidence linking cellular redox state to appropriate response to nutritional signals. J. Biol. Chem. 279:24387-24393.

Kim, H. J., Chen, C., Kabbage, M., and Dickman, M. B. 2011. Identification and characterization of Sclerotinia sclerotiorum NADPH oxidases. Appl. Environ. Microbiol. 77:7721-7729.

Krüger, A., Grüning, N. M., Wamelink, M. M., Kerick, M., Kirpy, A., Parkhomchuk, D., Bluemlein, K., Schweiger, M. R., Soldatov, A. Lehrach, H., Jakobs, C., and Ralser, M. 2011. The pentose phosphate pathway is a metabolic redox sensor and regulates transcription during the antioxidant response. Antioxid. Redox Signal. 15:311-324.

Kuge, S., Toda, T., Iizuka, N., and Nomoto, A. 1998. Crm1 (XpoI) dependent nuclear export of the budding yeast transcription factor yAP-1 is sensitive to oxidative stress. Genes Cells 3:521-532.
Lacaze, I., Lalucque, H., Siegmund, U., Silar, P., and Brun, S. 2015. Identification of NoxD/Pro41 as the homologue of the p22phox NADPH oxidase subunit in fungi. Mol. Microbiol. 95:1006-1024.

Lambeth, J. D. 2004. NOX enzymes and the biology of reactive oxygen. Nat. Rev. Immunol. 4:181-189.

Lara-Ortíz, T., Riveros-Rosas, H., and Aguirre, J. 2003. Reactive oxygen species generated by microbial NADPH oxidase NoxA regulate sexual development in Aspergillus nidulans. Mol. Microbiol. 50: $1241-1255$

Li, H., Zhang, Z., He, C., Qin, G., and Tian, S. 2016. Comparative proteomics reveals the potential targets of BcNoxR, a putative regulatory subunit of NADPH oxidase of Botrytis cinerea. Mol. Plant-Microbe Interact. 29:990-1003.

Malagnac, F., Lalucque, H., Lepère, G., and Silar, P. 2004. Two NADPH oxidase isoforms are required for sexual reproduction and ascospore germination in the filamentous fungus Podospora anserina. Fungal Genet. Biol. 41:982-997.

Marschall, R., and Tudzynski, P. 2016. BcIqg1, a fungal IQGAP homolog, interacts with NADPH oxidase, MAP kinase and calcium signaling proteins and regulates virulence and development in Botrytis cinerea. Mol. Microbiol. 101:281-298.

Minz Dub, A., Kokkelink, L., Tudzynski, B., Tudzynski, P., and Sharon, A 2013. Involvement of Botrytis cinerea small GTPases BcRAS1 and BcRAC in differentiation, virulence, and the cell cycle. Eukaryot. Cell 12:1609-1618.

Ngiam, C., Jeenes, D. J., Punt, P. J., Van Den Hondel, C. A., and Archer, D. B. 2000. Characterization of a foldase, protein disulfide isomerase A, in the protein secretory pathway of Aspergillus niger. Appl. Environ. Microbiol. 66:775-782.

Nowrousian, M., Frank, S., Koers, S., Strauch, P., Weitner, T., Ringelberg, C., Dunlap, J. C., Loros, J. J., and Kück, U. 2007. The novel ER membrane protein PRO41 is essential for sexual development in the filamentous fungus Sordaria macrospora. Mol. Microbiol. 64: 923-937.

O'Brien, J. A., Daudi, A., Butt, V. S., and Bolwell, G. P. 2012. Reactive oxygen species and their role in plant defence and cell wall metabolism. Planta 236:765-779.

Segmüller, N., Kokkelink, L., Giesbert, S., Odinius, D., van Kan, J., and Tudzynski, P. 2008. NADPH oxidases are involved in differentiation and pathogenicity in Botrytis cinerea. Mol. Plant-Microbe Interact. 21: 808-819.

Taylor, E. R., Hurrell, F., Shannon, R. J., Lin, T. K., Hirst, J., and Murphy, M. P. 2003. Reversible glutathionylation of complex I increases mitochondrial superoxide formation. J. Biol. Chem. 278:19603-19610.

Tian, S., Qin, G., and Li, B. 2013. Reactive oxygen species involved in regulating fruit senescence and fungal pathogenicity. Plant Mol. Biol 82:593-602.

Wang, L., Mogg, C., Walkowiak, S., Joshi, M., and Subramaniam, R. 2014. Characterization of NADPH oxidase genes NoxA and NoxB in Fusarium graminearum. Can. J. Plant Pathol. 36:12-21.

Wang, W., Cai, J., Wang, P., Tian, S., and Qin, G. 2017. Post-transcriptional regulation of fruit ripening and disease resistance in tomato by the vacuolar protease SIVPE3. Genome Biol. 18: Article 47.

Weiberg, A., Wang, M., Lin, F. M., Zhao, H., Zhang, Z., Kaloshian, I., Huang, H. D., and Jin, H. 2013. Fungal small RNAs suppress plant immunity by hijacking host RNA interference pathways. Science 342 : 118-123.

Williamson, B., Tudzynski, B., Tudzynski, P., and van Kan, J. A. 2007. Botrytis cinerea: The cause of grey mould disease. Mol. Plant Pathol. 8: 561-580.

Wiśniewski, J. R., Zougman, A., Nagaraj, N., and Mann, M. 2009. Universal sample preparation method for proteome analysis. Nat. Methods 6: 359-362.

Yang, S. L., and Chung, K. R. 2012. The NADPH oxidase-mediated production of hydrogen peroxide $\left(\mathrm{H}_{(2)} \mathrm{O}_{(2)}\right)$ and resistance to oxidative stress in the necrotrophic pathogen Alternaria alternata of citrus. Mol. Plant Pathol. 13:900-914. 